\title{
The relationship between lymphocyte subsets, nutritional status and tuberculin reactivity in continuous ambulatory peritoneal dialysis and hemodialysis patients
}

\author{
Mehmet Usta ${ }^{1} \cdot$ Alpaslan Ersoy $^{2} \cdot$ Yavuz Ayar $^{1} \cdot$ Ferrah Budak $^{3}$ \\ Received: 10 January 2020 / Accepted: 13 April 2020 / Published online: 22 April 2020 \\ (c) The Author(s) 2020
}

\begin{abstract}
Aim Skin test anergy is common in patients with uremia and during maintenance hemodialysis treatment. However, up to date only one study concerning skin test in peritoneal dialysis patients has focused on the issue. Our cross-sectional controlled study was conducted to analyze the correlation of purified protein derivative (PPD) test response with demographical features, nutritional parameters and the distribution of peripheral blood lymphocyte subsets in peritoneal dialysis and hemodialysis patients

Patients and methods Stable 30 hemodialysis (HD) patients (16 men, 14 women) and 30 continuous ambulatory peritoneal dialysis (PD) patients (17 men, 13 women) were included. Thirty healthy cases (15 men, 15 women) with a mean age of $32.4 \pm 9.4$ constituted the control group.

Results In the HD group, 14 patients (46.6\%) were PPD positive, and in the PD group 16 patients (53.3\%) were PPD positive. In the PPD-positive HD patients 64.2\% (9/14), and in the PPD-positive PD patients 62.4\% (10/16) had an induration of $10 \mathrm{~mm}$ or greater. In the control group, 21 of 30 patients (70\%) were PPD positive. Comparison of both HD and PD groups with the control group showed significant differences in PPD reactivity $(p<0.01)$. Albumin levels were significantly high in the control groups $(p<0.01)$, and cholesterol levels were significantly high in the PD and the control groups $(p<0.05)$. Transferrin levels were significantly high in the PD $(p<0.01)$. The lymphocyte counts were significantly high in the control group compared to the HD patients $(p<0.05)$. The lymphocyte subset percentages CD19 were high in the control groups $(p<0.05)$, and CD16/56 was significantly high in the PD groups $(p<0.05)$. All the parameters were also similar between PPD-positive and -negative same groups.

Conclusion The prevalence of PPD positivity was lower in the PD and HD groups. The PPD test responses were not related to the peripheral lymphocyte counts, subsets and malnutrition parameters.
\end{abstract}

Keywords Hemodialysis · Continuous ambulatory peritoneal dialysis · Tuberculin testing · Human erythropoietin · Nutritional status

Yavuz Ayar

yavuzayar@hotmail.com

1 Department of Nephrology, Bursa City Hospital, Nilüfer, 16010 Bursa, Turkey

2 Department of Nephrology, Faculty of Medicine, Uludag University, Bursa, Turkey

3 Department of Microbiology and Immunology, Faculty of Medicine, Uludag University, Bursa, Turkey

\section{Introduction}

It is known that an immune deficiency state is observed in chronic hemodialysis (HD) patients due to unknown mechanisms [1]. Both humoral and cellular immune systems have functional impairment in this setting. However, the major defect is generally detected in the cellular immune system in which T-lymphocytes have an important role [2]. Defective cell-mediated immunity, as reflected by anergy to multiple skin test antigens, is well documented in patients with protein-calorie malnutrition [3]. Skin test anergy is also common in patients with uremia and during maintenance hemodialysis treatment [4]. However, the reported prevalence 
varies widely. The mechanism leading to cutaneous anergy in chronic renal failure remains undefined. Various $\mathrm{T}$ cell abnormalities have been demonstrated, but the data are conflicting and there are almost equal numbers of reports of normal and depressed $\mathrm{T}$ cell function. The whole subset is beyond the scope of this communication [5-7]. In HD patients, decreased response to tuberculin, which is an anergic skin test, has been described. Simirnoff et al. reported an increased rate of anergy and tuberculin nonreactivity in this group of patients [8]. Recently, Yıldiz et al. reported the relationship between tuberculin response and patient demographic features, nutritional parameters, and the distribution of peripheral blood lymphocyte subgroups in HD patients [9]. In this report, they believed that the tuberculin response in hemodialysis patients could not be predicted by quantitative analysis of peripheral blood lymphocyte subtypes. The differences in tuberculin reactivity might originate from qualitative differences in lymphocyte subsets or differences in the dermal in situ immune response.

In the study, we aimed to investigate the association between tuberculin response and patient demographic features, nutritional parameters, and the distribution of peripheral blood lymphocyte subgroups in HD and PD.

\section{Patients and methods}

Stable 30 HD patients (16 men, 14 women) and $30 \mathrm{PD}$ patients (17 men, 13 women) were included in our study. The mean ages were $34.0 \pm 12.6$ and $34.4 \pm 12.6$ in the $\mathrm{HD}$ and PD patients, respectively. A control group of 30 healthy cases (15 men, 15 women) with a mean age of
$32.4 \pm 9.4$ years were also included. The demographic characteristics, state of erythropoietin therapy and total duration of dialysis are shown in Table 1. In chronic dialysis patients, no additional factors could affect the PPD results, except dialysis when present.

\section{Exclusion criteria}

- Presence of diabetes mellitus.

- History of tuberculosis infection.

- HIV positivity.

- Recent viral or bacterial infection.

- Having immunosuppressive therapy.

- A total dialysis duration of less than 6 months.

- Presence of malignancy.

- Age of less than 16 and older than 60 years.

In HD patients, during hemodialysis polysulfone membranes and bicarbonate dialysates were used. HD patients had two or three dialysis sessions per week. PD patients were dialyzed with standard peritoneal fluids containing $35 \mathrm{mmol} / \mathrm{L}$ lactate. Serum samples were taken just before the dialysis session and in the biochemistry and immunology laboratory of our center. The serum albumin, cholesterol and creatinine were determined by a standard autoanalyzer method. Serum transferrin was estimated by radial immunodiffusion (Nephelometers). Body mass indexes of all patients were determined. White blood cell count was determined by using Coulter counter and the lymphocyte count was derived from the differential count. PPD solutions were injected to both the patient and the control groups. $\mathrm{Kt} / V$ was taken as
Table 1 Demographic characteristics of the patients

\begin{tabular}{|c|c|c|c|c|c|c|}
\hline & \multicolumn{2}{|l|}{ Hemodialysis } & \multicolumn{2}{|c|}{ Peritoneal dialysis } & \multicolumn{2}{|l|}{ Control } \\
\hline & $\begin{array}{l}\text { PPD (+) } \\
n: 14\end{array}$ & $\begin{array}{l}\text { PPD (-) } \\
n: 16\end{array}$ & $\begin{array}{l}\text { PPD (+) } \\
n: 15\end{array}$ & $\begin{array}{l}\text { PPD (-) } \\
n: 15\end{array}$ & $\begin{array}{l}\text { PPD (+) } \\
n: 21\end{array}$ & $\begin{array}{l}\text { PPD (-) } \\
n: 9\end{array}$ \\
\hline Age (years) & $33.9 \pm 12.0$ & $34.1 \pm 11.8$ & $35.4 \pm 13.9$ & $33.5 \pm 13.4$ & $31.2 \pm 12.6$ & $33.6 \pm 10.9$ \\
\hline Sex (male/female) & $9 / 8$ & $7 / 6$ & $10 / 7$ & $7 / 6$ & $8 / 7$ & $7 / 8$ \\
\hline $\operatorname{BMI}\left(\mathrm{kg} / \mathrm{m}^{2}\right)$ & $22.1 \pm 3.2$ & $21.2 \pm 2.4$ & $23.0 \pm 3.0$ & $23.2 \pm 3.4$ & $24.0 \pm 2.3$ & $23.1 \pm 2.9$ \\
\hline TSD (months) & $30.8 \pm 13.8$ & $28.6 \pm 14.2$ & $27.0 \pm 12.7$ & $28.1 \pm 13.7$ & - & - \\
\hline Epo (UI/W) & $4386 \pm 3272$ & $4272 \pm 3849$ & $3872 \pm 3130$ & $3780 \pm 2964$ & - & - \\
\hline \multicolumn{7}{|c|}{ Primary etiology of renal failure } \\
\hline Idiopathic & 10 & & & 9 & & \\
\hline Hypertension & 5 & & & 6 & & \\
\hline GN & 6 & & & 4 & & \\
\hline Amyloidosis & 3 & & & 4 & & \\
\hline PKD & 2 & & & 3 & & \\
\hline ATN & 1 & & & 2 & & \\
\hline Pyelonephritis & 3 & & & 2 & & \\
\hline
\end{tabular}

ATN acute tubular necrosis, $P K D$ polycystic kidney disease, Epo erithropoietin, $W$ week, TSD time spent on dialysis, $G N$ glomerulonephritis 
the index of dialysis adequacy, and in the PD groups weekly $\mathrm{Kt} / V$ urea was calculated.

\section{Tuberculin testing}

Tuberculin reactivity in HD and PD patients was assessed by response to intradermal 5 IU PPD (Inter Vax Biologicals, Limited, Canada) injected using the Mantoux technique into the volar surface of the forearm and forearm without the arteriovenous fistula in the hemodialysis patients. Induration was measured at $72 \mathrm{~h}$. Indurations of less than $5 \mathrm{~mm}$ were defined as PPD negative in the dialysis patients. An experienced medical staff performed the tuberculin testing.

\section{Peripheral blood lymphocyte subtyping}

In the immunology laboratory, immunophenotypic analysis of the cells was performed using an EPICS XL-MCI flow cytometers (Coulter) equipped with a $15 \mathrm{~mW}$ air-cooled argon-ion laser. The subgroup of CD3, CD4, CD8, HLA $\mathrm{DR}^{+} \mathrm{CD}^{+}, \mathrm{CD} 16 / 56^{+}$and CD19 lymphocytes were measured by using monoclonal antibodies in peripheral blood samples. A minimum of 1000 events was counted on each sample. Data analysis was performed using EPICS XL-MCI software (Coulter). Gating was performed using $90^{\circ}$ right angle scatter. The fluorescence signals were amplified on a logarithmic scale.

\section{Statistical analysis}

We reported all numerical values as mean \pm standard deviation (SD). Statistical computations were done using SPSS for Windows V. 20.0 (SPSS Inc. Illinois, USA). Using the nonparametric Kruskal-Wallis ANOVA test, we did comparisons between groups. Statistical significance was assumed for $p$ values less than 0.05 .

\section{Results}

The comparison of both dialysis groups revealed no significant differences in mean ages, sex, body mass indexes, and total duration of dialysis $(p>0.05)$. In addition, comparison of dialysis patients with the control group revealed no significant differences in characteristics of patients $(p>0.05)$. Distributions of dialysis patients according to primary etiologies were similar (Table 1).

The mean durations of HD in PPD-positive and -negative patients were $30.7 \pm 13.8$ and $28.6 \pm 14.2$ months, respectively $(p>0.05)$. In the PD group, the mean duration of dialysis was $27.0 \pm 12.7$ and $28.1 \pm 13.7$, in the PPD-positive and -negative patients, respectively $(p>0.05)$. In all dialysis groups, erythropoietin dosages did not differ significantly $(p>0.05)$.

In the HD group, 14 patients (46.6\%) were PPD positive and 16 (53.3\%) were PPD negative. In the PD group, 16 patients $(53.3 \%)$ were PPD positive and 14 (46.6\%) were negative. In the PPD-positive HD patients 64.2\% (9/14) and in the PPD-positive PD patients $62.4 \%$ (10/16) had an induration of $10 \mathrm{~mm}$ or greater. In the control group, 21 of 30 patients $(70 \%)$ were PPD positive. The mean PPD values are demonstrated in Table 2. The PPD response of the two patient groups were compared and no statistical significance was found $(p>0.05)$. However, comparison of both HD and PD groups with the control group showed significant differences in PPD reactivity $(p<0.01)$ (Table 2$)$.

Serum creatinine levels were determined in all dialysis patients with a $\mathrm{Kt} / V$ of greater than 1.7 and in the PPDpositive and -negative dialysis patients no significant intraand intergroup differences were demonstrated $(p>0.05)$. Albumin levels did not significantly differ in the dialysis groups and intergroup comparisons revealed no significant differences $(p>0.05)$. In the control group, albumin levels were significantly high compared to the dialysis groups $(p<0.05)$. Serum cholesterol levels were significantly high in the PPD-positive and -negative control groups and the PPD-positive and -negative PD groups compared to the HD groups $(p<0.05)$. The serum cholesterol levels were uniformly distributed in all groups ( $p>0.05$, Table 3$)$. Transferrin level was higher in PD than HD $(p<0.01)$; however, intergroup comparisons revealed no significant differences $(p>0.05)$.

In all groups, peripheral lymphocyte counts and subgroups were evaluated. There was no a significant difference between the lymphocyte counts of the HD and PD groups $(p>0.05)$. The lymphocyte counts were significantly higher in the control groups compared to the HD patients $(p<0.05)$. There was no significant difference between those of control and PD groups $(p>0.05)$. The lymphocyte subsets CD3, $\mathrm{CD} 4, \mathrm{CD} 8$, and CD4/CD8 did not change significantly among all groups $(p>0.05)$. However, the lymphocyte subset CD19 was significantly higher in the PPD-positive and -negative control groups compared to both dialysis groups (c: $p<0.05, \mathrm{~d}: p>0.01$, Table 4 ). The subset CD16/56 was significantly higher in PPD-positive and -negative PD groups compared to the control groups and HD groups $(p<0.05)$.

Table 2 PPD values and response rates of the groups

\begin{tabular}{llll}
\hline & HD & PD & Control \\
\hline PPD (+) (\%) & 46.6 & 53.3 & 70 \\
PPD (mm) & $7.6 \pm 5.7$ & $8.2 \pm 6.0$ & $16.7 \pm 4.8^{\mathrm{a}}$ \\
\hline${ }^{\mathrm{a}} p<0.01$, both dialysis groups were compared to the control group
\end{tabular}


Table 3 Nutritional parameters of the patients

\begin{tabular}{lccccll}
\hline & PPD (+)HDp & PPD (-) HDp & PPD (+)PDp & PPD (-)PDp & PPD $(+) C$ & PPD $(-) C$ \\
\hline Creatinine (mg/dL) & $10.7 \pm 2.8$ & $12.2 \pm 2.0$ & $11.0 \pm 2.7$ & $11.4 \pm 3.2$ & - & - \\
Albumin (g/dL) & $3.6 \pm 0.4$ & $3.7 \pm 0.2$ & $3.5 \pm 0.3$ & $3.6 \pm 0.3$ & $4.0 \pm 0.3^{\mathrm{a}}$ & $4.1 \pm 0.4^{\mathrm{a}}$ \\
Cholesterol (mg/dL) & $151 \pm 36$ & $158 \pm 47$ & $208 \pm 37^{\mathrm{b}}$ & $183 \pm 30^{\mathrm{b}}$ & $188 \pm 49^{\mathrm{b}}$ & $203 \pm 32^{\mathrm{b}}$ \\
Transferrin (mg/dL) & $1.61 \pm 0.3$ & $1.58 \pm 0.3$ & $1.92 \pm 0.4^{\mathrm{c}}$ & $1.82 \pm 0.4^{\mathrm{c}}$ & - & - \\
\hline
\end{tabular}

$H D p$ hemodialysis patients, $P D p$ peritoneal dialysis patients, $C$ control

${ }^{a} p<0.01$, compared to the PPD-positive and -negative dialysis groups

${ }^{\mathrm{b}} p<0.01$, compared to the PPD-positive and -negative HD groups

${ }^{c} p<0.01$, compared to the PPD-positive and -negative HD groups

Table 4 Peripheral lymphocyte counts and subgroups

\begin{tabular}{|c|c|c|c|c|c|c|}
\hline & HDp, PPD (+) & HDp, PPD (-) & PDp, PPD (+) & PDp, PPD (-) & $\mathrm{C}, \mathrm{PPD}(+)$ & C, PPD (-) \\
\hline $\mathrm{PBL}, / \mathrm{mm}^{3}$ & $1518 \pm 718$ & $1535 \pm 634$ & $1787 \pm 653$ & $1826 \pm 679$ & $2105 \pm 458^{\mathrm{a}}$ & $2090 \pm 409^{\mathrm{a}}$ \\
\hline $\mathrm{CD} 3, \%$ & $73.2 \pm 8.9$ & $73.3 \pm 8.2$ & $68.8 \pm 8.7$ & $72.3 \pm 6.3$ & $72.9 \pm 5.9$ & $70.6 \pm 4.3$ \\
\hline $\mathrm{CD} 4, \%$ & $44.1 \pm 8.3$ & $42.8 \pm 6.1$ & $45.2 \pm 4.9$ & $48.0 \pm 5.2$ & $46.4 \pm 7.3$ & $42.0 \pm 5.4$ \\
\hline $\mathrm{CD} 8, \%$ & $30.3 \pm 7.8$ & $30.2 \pm 5.8$ & $25.5 \pm 7.4$ & $26.1 \pm 5.9$ & $25.5 \pm 4.6$ & $25.8 \pm 6.0$ \\
\hline CD4/CD8 & $1.6 \pm 0.6$ & $1.4 \pm 0.3$ & $1.9 \pm 0.5$ & $1.9 \pm 0.4$ & $1.8 \pm 0.5$ & $1.7 \pm 0.5$ \\
\hline CD19, \% & $8.4 \pm 3.1$ & $8.2 \pm 1.9$ & $8.9 \pm 5.0$ & $8.5 \pm 4.1$ & $11.5 \pm 3.9^{b}$ & $13.4 \pm 5.6^{\mathrm{c}}$ \\
\hline $\mathrm{CD} 16^{+} / 56^{+}, \%$ & $7.6 \pm 4.2$ & $8.0 \pm 5.7$ & $13.5 \pm 7.8^{\mathrm{d}}$ & $13.9 \pm 6.6^{\mathrm{d}}$ & $7.2 \pm 4.0$ & $6.3 \pm 3.0$ \\
\hline $\mathrm{HLA} \mathrm{DR}^{+} \mathrm{CD}^{+}, \%$ & $25.2 \pm 12.8$ & $21.5 \pm 9.1$ & $26.3 \pm 9.9$ & $19.9 \pm 8.1$ & $19.9 \pm 5.8$ & $22.6 \pm 9.9$ \\
\hline
\end{tabular}

$P B L$ periheral blood lymphocyte, $H D p$ hemodialysis patients, $P D p$ peritoneal dialysis patients, $C$ control

${ }^{\mathrm{a}} p<0.05$, compared to the PPD-positive and -negative HDp groups

${ }^{\mathrm{b}} p<0.05, \mathrm{PPD}(+) \mathrm{C}$ group was compared to the PPD-positive and -negative HDp and PDp groups

${ }^{c} p<0.01$, PPD(-) C group was compared to the PPD-positive and -negative HDp and PDp groups

${ }^{\mathrm{d}} p<0.05$, compared to the PPD-positive and -negative $\mathrm{C}$ groups and HDp groups

\section{Discussion}

Delayed skin hypersensitivity was clearly depressed, out of proportion to the number of patients manifesting protein-calorie malnutrition. Protein-calorie malnutrition had been described in the chronic hemodialysis population. Positive skin tests were seen in $36-50 \%$ of the uremic patients. They concluded that the incidence of anergy increased with the duration of dialysis. Delayed cutaneous hypersensitivity to microbial or protein antigens is commonly depressed in uremic patients (50-60\%) compared to healthy controls $[1,3-5,10]$. In our study, we found that the prevalence of PPD positivity in chronic HD and PD patients was $\% 46.6$ and \%53.3, respectively. In PPDpositive HD patients $64.2 \%$ (9/14), and in PPD-positive PD patients \% $62.4(10 / 16)$ had an induration of $10 \mathrm{~mm}$ or more. The PPD positivity rates of these two groups were similar as well as the total dialysis duration. However, both the HD and PD groups had significantly lower PPD positivity rates compared to the control groups. Studies concerning immune system status, nutrition and dermal anergy tests are frequently held in hemodialysis groups, and not much data are available for PD patients. Taskapan et al. evaluated 30 PD patients and found that $20 \%$ had indurations of $5 \mathrm{~mm}$ or more in dermal PPD anergy test [7]. In our study, serum albumin and creatinine levels, which are important parameters for malnutrition, were similar in PPD-positive and -negative HD and PD groups. Serum cholesterol levels were significantly high in both the PPD-positive and -negative PD groups. The high levels in the PD group may be due to high glucose content in the dialysate solutions and better nutritional status in peritoneal dialysis patients in contrast to other studies. The prevalence of malnutrition in PD patients is greater than that in HD patients. A recent international multicenter study found a prevalence of moderate to severe malnutrition in $40 \%$ of these patients $[11,12]$. In our study, PPD positivity, albumin and creatinine levels and BMI were similar in the PPD-positive and -negative HD and PD groups. In the control group in PPD-positive and -negative patients, serum albumin levels were significantly high. Albumin is a positive marker for malnutrition, but we considered the high levels of serum albumin to be independent in the PPD-positive control group. Additionally, transferrin that is a nutritional parameter was significantly higher in 
the PD group. There was no difference in both groups in terms of total lymphocyte count, and PPD positivity and total lymphocyte count were significantly higher in the control group compared to PD and HD patients. Iron overload, due to changes in its levels in proteinuric patients and being an acute phase reactant, is not accepted as an optimal nutritional parameter. Rapid turnover of iron may helps short-term evaluation of nutritional status. In the study ion dialysis patients, the measured PPD reactivity was significantly low. The mechanism leading to cutaneous anergy in chronic renal failure remains undefined. Various $\mathrm{T}$ cell abnormalities have been demonstrated, but the data are conflicting and there are almost equal numbers of reports of normal and depressed $\mathrm{T}$ cell function. Uremic patients are always profoundly lymphopenic [5, 6, 13]. Significantly low absolute counts of the total population of $\mathrm{T}$ cells as well as $\mathrm{T}$ cell regulatory subsets and $\mathrm{B}$ cells have been described [14]. Uek1 et al. reported that the numbers of peripheral blood lymphocyte, CD3, CD4, CD8, and CD20 cells were decreased in HD patients compared to those in healthy subjects, while the number of CD3 HLA-DR cell was increased in HD patients compared to that in healthy subjects. In this study, they documented that HLA DR CD3 levels decreased after 1 month of erythropoietin therapy and gradually declined throughout the 6-month study period [15]. In the PD patients, the previously mentioned parameters are not fully evaluated and not much data comparing PPD-positive and -negative HD and PD groups are available. In our study, the total lymphocyte counts were similar in the PPD-positive and -negative HD and PD groups. However, in the PPD-positive and -negative HD patients, the total lymphocyte counts were significantly lower than that in the PPD-positive and -negative control group. The total lymphocyte counts did not differ between the PPD-positive and -negative groups in the dialysis patients. In addition, in the PD group the total lymphocyte counts were a little higher in the PPDpositive patients. In the control group, high blood lymphocyte count may be associated with PPD positivity. Impaired immune response in uremia may be caused by multiple derangements of the immune system. In another investigation evaluating the lymphocyte subsets. Deenitchina et al. reported that CD3 and CD4 percentage rate was significantly high in HD patients than that in the control group [1]. In another study conducted by Yildiz et al., 29 HD patients were studied and the HLA DR CD3 percentage rate was significantly high in the PPD-positive and -negative HD group than that of the healthy controls. CD3, CD4, CD8, CD4/CD8, CD19 and CD16/56 percentage rated were similar [9]. In our study, the CD16/56 lymphocyte subsets were significantly more frequent in the PPD-positive and -negative PD groups than that of the PPD-positive and -negative HD and the control patients.
CD19 percentage rate was significantly high in the control group compared to both dialysis groups. The percentages of subgroup of CD3, CD4, CD8 lymphocytes, and the $\mathrm{CD} 4 / \mathrm{CD} 8$ ratio were similar in all groups. The results of our study are in concordance with the ones regarding the HD population, but in PD patients the data on these subsets are not much. In a study, a lowering effect of erythropoietin in lymphocyte subsets of HLA DR CD3 in HD patients was demonstrated [15]. In our study, we did not have data encouraging us to think erythropoietin causes a decrease in lymphocyte subsets of HLA DR CD3. In our study, the distribution of lymphocyte subsets was similar in dialysis patients receiving the same amount of erythropoietin, except for the high prevalence of CD16/56 ratio in PPD-positive and -negative PD patients. The subsets were also similar in the control group, except for the high prevalence of lymphocyte subgroup CD19. In general studies, lymphocyte subgroups are expected to be lower in dialysis patients compared to healthy individuals $[1,9,15]$. In our study, CD19 levels were higher in the control group.

In conclusion, nutritional status, PPD results, and peripheral lymphocyte counts were similar in PPD-positive and -negative patients in all dialysis groups. Lymphocyte subset CD19 was more prevalent in the control group and CD16/56 ratio was more prevalent in the PD group. The other lymphocyte subset distributions did not differ significantly among all dialysis groups and the controls. In addition, PPD skin anergy test and serum albumin levels were high in the control groups compared to the dialysis groups. In this study, though the lymphocyte counts were similar in PPD-positive and -negative PD and the control groups, PPD positivity was significantly high in the control group. This may be due to a lymphocyte function abnormality in the PD patients, as reported in HD patients previously $[16,17]$. Another interesting point is the similarity of malnutrition parameters, lymphocyte counts and subsets in all same groups between PPD-positive and -negative patients, suggesting that other possible factors influencing the PPD results may exist. Nutritional status, immunity and body resistance, and personal characteristics of dialysis patients (history of disease, etc.) increase the tendency toward infection and may affect the PPD results [18-20]. Our study supports these data. There is no difference in tuberculin and qualitative tests in terms of PPD positivity and negativity for both dialysis types. We need larger studies. In addition, though dialysis patients have an increased risk of mycobacterium infection, PPD skin test is not frequently reactive and cannot rule out a tuberculosis infection.

Open Access This article is licensed under a Creative Commons Attribution 4.0 International License, which permits use, sharing, adaptation, distribution and reproduction in any medium or format, as long as you give appropriate credit to the original author(s) and the source, provide a link to the Creative Commons licence, and indicate if changes were made. The images or other third party material in this article are 
included in the article's Creative Commons licence, unless indicated otherwise in a credit line to the material. If material is not included in the article's Creative Commons licence and your intended use is not permitted by statutory regulation or exceeds the permitted use, you will need to obtain permission directly from the copyright holder. To view a copy of this licence, visit http://creativecommons.org/licenses/by/4.0/.

\section{References}

1. Deenitchina SS, Ando T, Okuda S, Kinukawa N, Hirakata H, Nagashima A, Fujishima M (1995) Cellular immunity in hemodialysis patients: a quantitative analysis of immune cell subsets by flow cytometry. Am J Nephrol 15(1):57-65

2. Chiu YL, Shu KH, Yang FJ, Chou TY, Chen PM, Lay FY, Pan SY et al (2018) A comprehensive characterization of aggravated aging-related changes in T lymphocytes and monocytes in endstage renal disease: the iESRD study. Immun Ageing. 15:27. https ://doi.org/10.1186/s12979-018-0131-x (ECollection 2018)

3. Bahwere P, James P, Abdissa A, Getu Y, Getnet Y, Sadler K, Girma T (2017) Use of tuberculin skin test for assessment of immune recovery among previously malnourished children in Ethiopia. BMC Res Notes 10(1):570. https://doi.org/10.1186/ s13104-017-2909-X

4. Deniz S, Aydemir Y, Şengül A, Emre JÇ, Tanrisev M, Özhan MH, Güney İ (2019) Factors affecting TST level in patients undergoing dialysis: a multicenter study. Hemodial Int. 23(1):81-87. https:// doi.org/10.1111/hdi.12676

5. Myall K, Milburn HJ (2017) An update on the management of latent tuberculosis infection and active disease in patients with chronic kidney disease. Pol Arch Intern Med 127(10):681-686

6. Chagas AC, Hans Filho G, de Oliveira SM, Ivo ML, Corrêa Filho RA, Donatti MI (2014) Prevalence of latent tuberculosis and treatment adherence among patients with chronic kidney disease in Campo Grande, State of Mato Grosso do Sul. Rev Soc Bras Med Trop 47(2):204-211

7. Taskapan H, Oymak O, Utas C (2000) Tuberculin and anergy testing in CAPD patients. Perit Dial Int 20(6):807-809

8. Smirnoff M, Patt C, Seckler B, Adler JJ (1998) Tuberculin and anergy skin testing of patients receiving long-term hemodialysis. Chest 113(1):25-27

9. Yildiz A, Akkaya V, Yildiz P, Tûrk S, Görel N, Sever MS, Badur $S$ (1999) No relation of tuberculin reactivity with quantitative analysis of peripheral blood lymphocyte subsets in haemodialysis patients. Respir Med 93(2):119-122
10. Campbell JR, Johnston JC, Ronald LA, Sadatsafavi M, Balshaw RF, Cook VJ, Levin A, Marra F (2019) Screening for latent tuberculosis infection in migrants with CKD: a cost-effectiveness analysis. Am J Kidney Dis 73(1):39-50

11. Johansson L (2015) Nutrition in older adults on peritoneal dialysis. Perit Dial Int 35(6):655-658

12. Günalay S, Öztürk YK, Akar H, Mergen H (2018) The relationship between malnutrition and quality of life in haemodialysis and peritoneal dialysis patients. Rev Assoc Med Bras (1992) 64(9):845-852

13. Revillard JP (1997) Interview with Jean-Pierre Revillard. What is going to happen tomorrow as far as treatment of autoimmune disorders is concerned? Ann Med Interne (Paris) 148(1):11-14

14. Aly MG, Zhu L, Weimer R, Opelz G, Morath C, Kuon R, Tohamy $M$ et al (2017) Low utility of serum 25-hydroxyvitamin D3 and 1, 25-dihydroxyvitamin D3 in predicting peripheral Treg and Th17 cell counts in ESRD and renal transplant patients. Transpl Immunol 43-44:3-10

15. Ueki Y, Nagata M, Miyake S, Tominaga Y (1993) Lymphocyte subsets in hemodialysis patients treated with recombinant human erythropoietin. J Clin Immunol 13(4):279-287

16. Griveas I, Visvardis G, Fleva A, Papadopoulou D, Mitsopoulos E, Nikopoulos K, Manou E et al (2004) Lymphocytes subsets in the course of continuous ambulatory peritoneal dialysis (CAPD). Ren Fail 26(6):641-646

17. Grzegorzewska AE, Leander M (2000) Total lymphocyte count during the course of CAPD treatment. Perit Dial Int 20(5):577-579

18. Jebali H, Barrah S, Rais L, Kheder R, Khouja N, Mhiri SN, Beji M, Abdelmalek R (2017) The diagnosis of tuberculosis in dialysis patients. Saudi J Kidney Dis Transpl 28(6):1362-1368

19. Unsal A, Ahbap E, Basturk T, Koc Y, Sakaci T, Sinangil Arar A, Kayabasi H, Sevinc M (2013) Tuberculosis in dialysis patients: a nine-year retrospective analysis. J Infect Dev Ctries 7(3):208-213

20. Ferguson TW, Tangri N, Macdonald K, Hiebert B, Rigatto C, Sood MM, Shaw $S$ et al (2015) The diagnostic accuracy of tests for latent tuberculosis infection in hemodialysis patients: a systematic review and meta-analysis. Transplantation 99(5):1084-1091

Publisher's Note Springer Nature remains neutral with regard to jurisdictional claims in published maps and institutional affiliations. 